\title{
A Study of Recommender Systems on Social Networks and Content-based Web Systems
}

\author{
Rahul Hooda \\ M.Tech. (Software Engineering) \\ U.I.E.T, Kurukshetra University, \\ Kurukshetra, Haryana, India
}

\author{
Kulvinder Singh \\ Assistant Professor \\ U.I.E.T, Kurukshetra University, \\ Kurukshetra, Haryana, India
}

\author{
Sanjeev Dhawan \\ Assistant Professor \\ U.I.E.T, Kurukshetra University, \\ Kurukshetra, Haryana, India
}

\begin{abstract}
Everybody rely on recommendations in everyday life from other people either orally or by reviews printed in newspapers or websites. Recommender systems are a subfamily of information filtering systems that explore to predict the 'rating' or 'preference' that user would give to an item. These systems are best known for their use in e-commerce websites where they use input about a customer's interest to generate a list of recommended items. Many recommender systems explicitly rate to represent customer's interest by using only the items that the customers purchase, but can also use other attributes, including items viewed, subject interests and demographic data. They direct users towards those items that meet their needs by reducing unwanted information spaces. To perform recommendation a number of techniques have been proposed, including content-based, collaborative, and hybrid techniques. To improve performance and to outweigh the drawbacks of individual recommendation techniques, these techniques are sometimes combined to form hybrid recommenders. This paper is categorized into seven sections. Section-I presents the introduction related to the recommendation systems used in the social networks and online Web systems, section-II critically analyzed the related literature work about collaborative recommendation, contentbased recommendation, and hybrid recommendation, sectionIII describes the business aspects of recommender systems, section-IV describes various ways of displaying recommendations to a customer, section- $\mathrm{V}$ investigates the various recommendations techniques and their limitations and section-VI provides the conclusion of the recommender systems. In this paper the efforts are made to review and discover the techniques to investigate the proper usage of recommender systems in the e-commerce applications.
\end{abstract}

\section{Keywords}

Electronic commerce, recommender system, collaborative recommendation, content-based recommendation, hybrid recommendation

\section{INTRODUCTION}

Mostly all large-scale commercial and social websites recommend products, people or links to connect with, to users. Recommendation systems traverse massive amounts of data to identify potential user preferences. Recommendation systems changed the way quiescent websites communicate with their users. Websites today rather than providing a static experience in which users search for and buy products, are more interactive. Recommendation systems increase interaction to provide a richer experience. Recommender systems are used in many websites you use every day including LinkedIn, Amazon, Hulu, and Netflix. LinkedIn a business-oriented social networking website is used to find jobs and business opportunities suggested by someone in one's contact network. LinkedIn recommender uses profile data to suggest top jobs to their members. 'Talent Match' feature of LinkedIn recommender suggests top candidates for the job in real time whenever a recruiter posts jobs. LinkedIn recommender uses a combination of content matching and collaborative filtering to suggest companies, a user might be interested in Amazon one of the most popular e-commerce websites, makes heavy use of an item to item collaborative filtering. For each item, Amazon builds a neighborhood of similar items. Whenever one buy/look at an item, Amazon then recommends items from that item's neighborhood. Video-streaming website Hulu, uses a recommender system to suggest videos that might be of interest to their users. Hulu also uses item to item collaborative filtering as their basic recommendation algorithm. Netflix, the video rental and streaming service uses collaborative filtering algorithms to show recommendations. Netflix held a competition to improve its recommendation system. The algorithms developed as part of the competition are mix of a large number of different machine learning techniques. To perform collaborative filtering, matrix factorization and temporal dynamics were the two most prominent features that emerged from the competition. Reel.com recommendations are based on individual's patterns of purchases. Consider a group of people who like the movies you like and dislike the movies you dislike. Some members of this group will have seen and liked movies that you have not yet seen. Reel.com recommender system would suggest those movies to you. Jester, a joke recommender system uses Eigentaste, a collaborative filtering algorithm to recommend jokes to a user based on user ratings of previous jokes. YouTube's recommendation systems bring videos to a user that it believes the user will be interested in. To increase the duration of time the user spends on the website, YouTube's recommender system coalesces the related videos association rules with the users personal actions on the website. "Just for You" feature of iTunes music store from apple recommends songs and albums to a user. TripAdvisor.com recommender suggests lodging and places to visit.

\section{RELATED WORK}

\subsection{Collaborative Recommendation}

There exists many collaborative recommender systems in the academe and in the industry. Grundy system presented by E. Rich (1979) [1], a librarian program was the first recommender system that proposed to build models of users based on a limited amount of information on each individuals 
using stereotypes. It gathers personal information through interactive dialogue, matches user responses against a database (library) of user stereotypes and recommends books. Tapestry [2], a manual collaborative filtering system presented by Goldberg et al. relied on each user to identify like-minded users manually. It was designed to recommend documents drawn from newsgroups to a group of users. GroupLens [3,4], Video Recommender [5], and Ringo [6] were the first automated collaborative filtering system .

The GroupLens [3,4] system was also developed for filtering text documents (i.e., news articles), but was designed for use in an open community and introduced the basic idea of automatically finding similar users in the database for making predictions. The Ringo [6] system, presented by Shardanand and Maes (1995) describes a music recommender based on collaborative filtering using Pearson's correlation measure and the mean absolute error (MAE) evaluation metric. Other examples of collaborative recommender systems include the Amazon.com book recommendation system and the Jester system [7] that recommends jokes.

According to Breese et al. [8], algorithms for collaborative recommendations can be grouped into two general classes: model-based and memory-based. Memory-based algorithms $[4,8,9,10]$ are heuristics that use previously rated items by the users to make rating predictions. That is, the value of the unknown rating $r_{c, x}$ for item $x$ and user $\mathrm{c}$ is usually computed as an aggregate of the ratings of some other (usually the $\mathrm{N}$ most similar) users for the same items:

$$
\begin{aligned}
& r_{c, x}=\operatorname{aggr} r_{c^{\prime}, x} \\
& c^{\prime} \epsilon \hat{C}
\end{aligned}
$$

Where $\hat{C}$ indicates the array of $\mathrm{N}$ users who have rated item $\mathrm{s}$ and are most similar to user c. In contrast to memory-based methods, model-based algorithms [7,8,10,11,12,13,14] first uses a set of ratings to learn a model, then using this model make rating predictions. For example, probabilistic approach proposed by Breese et al. [8] to collaborative filtering, where the unknown ratings are calculated as

$$
r_{c, x}=\sum_{i=0}^{n} i * \operatorname{Pr}\left(r_{c, x}=i \mid r_{c, x}, \epsilon X_{c}\right)
$$

It is assumed that the rating values are integers between 0 and $n$. The probability expression states the probability that user $\mathrm{c}$ will give a rating to item $\mathrm{x}$, given the users ratings of the previously rated items. Breese et al. proposes two probabilistic models to estimate this probability namely: Bayesian belief networks and cluster models. In cluster model, like-minded users are grouped into clusters. The user ratings are assumed to be independent (given the user class membership), a prediction for a certain user $u$ and an item $i$ can be made based on the probability of user $u$ falling into a certain cluster and the probability of liking item $i$ when being in a certain cluster given the user's ratings. The parameters for the model and estimate of number of clusters to use can be determined by using standard techniques such as expectation maximization algorithm [15]. In Bayesian model, each item in the domain is represented as a node in a Bayesian network. These networks allow us to make existing dependencies between variables explicit and encode these relationships in a directed acyclic graph. The states of each node in the network correspond to the possible rating values for each item. Model building requires the system to learn structure of network and the required conditional probabilities.

\subsection{Content-based Recommendation}

G. Salton, in book "Automatic Text Processing" [16] has shown that the content-based recommendation has its roots in information retrieval and information filtering. It is due to the early advancements made by the information retrieval and filtering communities that many content-based recommenders focus on recommending items containing textual information such as URLs and documents. Fab system [17] presented by Balabanovic \& Shoham (1997) recommended Web pages to users, presented 100 most important keywords in the content along with the web page content. Similarly, the Syskill \& Webert system [18] presented by Billsus \& Pazzani, represented documents with the 128 most informative words. There are several methods that could determine the "importance" of word in document such as Dice coefficient, probabilistic methods and explicit decision models. One of the best-known measures for specifying keyword weights is the term frequency/inverse document frequency (TF-IDF) measure [16] used by G Salton (1989). Content-based systems recommend those items that are similar to those liked by the user in the past. Pazzani \& Billsus [18] used a Bayesian classifier in order to estimate the probability that a document will be liked. NewsDude [19] presented by Billsus \& Pazzani (1999), a content-based filtering system suggests new stories the user might like to read. To accomplish this two user models are built. The first user model measure similarity between the new story and the stories that the user has read before by counting the co-occurrences of words appearing in these stories. The second user model assigns a probability of interest to a new story by comparing how frequently its words occur in those stories the user regards as interesting to those the user regards as of no interest.

\subsection{Hybrid Recommendation}

One way to build hybrid recommender systems is to implement separate collaborative and content-based systems and then combine the outputs (ratings) obtained from individual recommender systems into one final recommendation. Daily Learner system [20] presented by Billsus \& Pazzani (2000), selected the recommender system that gave the recommendation with the higher level of confidence, while hybrid recommender system presented by Tran \& Cohen [21] chose the one whose recommendations were more consistent with past ratings of the user. Many hybrid recommender systems, including the "collaboration via content" approach described by M. Pazzani (1999) [22] and the Fab system [17], were based on traditional collaborative techniques but also maintained the content-based profiles for each user. Fab system [17] used content-based filtering, which ranked documents and considered user's feedback to update their personal selection agent's profile. As presented by M. Pazzani [22], this allows to overcome some sparsity-related problems of a purely collaborative approach, since typically not many users will have a substantial number of commonly rated items. Basu et al. (1998) [23] proposed to use contentbased and collaborative characteristics (e.g., the age or gender of users or the genre of movies) in a single rule-based classifier. Popescul et al. [24] and Schein et al. [25] proposed a unified probabilistic method for combining collaborative and content-based recommendations. Several papers, such as $[17,22,26,27]$, states that hybrid approaches provide more accurate recommendations than traditional approaches by empirically comparing the performance of hybrid with collaborative and content-based approaches. 


\section{BUSINESS CHARACTERISTICS OF RECOMMENDER SYSTEMS}

Since e-commerce companies have plunged into ever increasing competition, so companies are always on the lookout for ways to increase their sales figures. Since businesses typically have large amount of products for sale, therefore the use of recommender systems in an e-commerce environment improve the financial performance by helping customers find the right product. As explained by Schafer et al. [28] recommender systems can enhance e-commerce dialogues in the following ways:

\subsection{Turning Browsers into Buyers}

Recommender systems aim to increase loyalty on e-commerce websites, convert browsers into buyers by recommending items that best fit their interests; these may include unplanned purchases driven by serendipity from the recommendations made.

\subsection{By Increasing Cross-sell}

Recommender systems improve cross selling by suggesting additional items associated with the products in the shopping cart before the checkout process. This brings the customer back to the shelves and thus increases the average order size.

\subsection{By Building Loyalty}

In the Internet the competitor is always just one click away, building customer-loyalty becomes an essential aspect of business strategy. Loyalty can be defined as the intention to repurchase products or services by a customer through a particular e-service vendor. Recommender systems improve loyalty by creating a relationship between the site and the customer. The system learns more about a customer's preferences and interests, each time the customer visits the website. The system utilizes this information to personalize what is offered. By providing each customer with a personalized experience, a parallel improvement in the likelihood of that customer returning is achieved.

\subsection{By Providing Herd Mentality}

Customers tend to flock to where other customers are hovering. For example if there is a property along the national highway and some people seem curious about it, then it must be a good value, location or both. Other potential customers will likely become interested too. Recommender system provides herd mentality by displaying number of people hovering or seeing a particular product.

\subsection{By Improving Up-sell}

Up-selling means recommending a similar but more expensive product to the customer. For example, if a customer is looking for $8 \mathrm{~GB}$ pendrive, offer him $16 \mathrm{~GB}$ at a discount.

\section{HOW TO PROVIDE RECOMMENDATIONS}

There are many ways to display recommendations to a customer. The method selected may well depend on how the e-commerce site wants the customer to use the recommendation. Here we will examine several recommendation interfaces. Each of them draws upon the strengths of the electronic medium to provide more powerful recommendations.

\subsection{Display Similar Items}

Similar item recommendation is one alteration from traditional techniques which large e-commerce websites often implement because the need to scan a vast number of potential neighbors (in case of collaborative recommendation) makes it impossible to compute predictions in real time. The idea behind the item based recommendations is to compute the predictions using similarity between items and not between users. The items displayed can be selected on the basis of items in which a customer has shown interest. Thus sites increase customer's exposure to their product line, improves up-sell and are able to sell more items per order.

\subsection{Email}

Recommendations can also be delivered directly to customers through email, in an extension of traditional direct mail techniques. According to survey conducted by listark.com [29], online shoppers would like to receive emails featuring merchandise selected based on their own shopping habits. Shoppers are ready to share more information with retailers in order to receive emails that are relevant to them. They are also happy to receive more emails if the emails have products based on their own preferences and shopping habits.

\subsection{Text Comments}

Progressively, text comments given by customers for products are being used by websites to provide customers with recommendations. Amazon.com, Flipkart.com, Snapdeal.com customer comments and eBay's feedback profile simplify the gathering of "the word on the street" by allowing customers to find an item of interest and browse the comments of other customers for that item. This helps sites make money by providing confidence on the goods/services being sold - the thought being, if sufficient people claim that a product is good and the seller is credible, than it is likely to be true. This not only helps convert browsers into buyers, but also increase loyalty to a site. If customers learn they can trust these third party recommendations, than they are more likely to return the next time they are faced with a questionable decision. According to "Local Consumer Survey" by Myles Anderson [30], online reviews, ratings and opinions left by consumers are playing an ever increasing role in helping other as anonymous consumers asses the quality \& trustworthiness of businesses, products and services. Browsers becomes buyers as online customer reviews put rich and subjective information about the product of interest. They enable people to prejudge a business to deciding on which business/product to buy for themselves.

\subsection{Average Rating}

Average rating on a product closely associate with conversion to purchase. Rather than providing text based reviews, customers can provide numerical ranking opinions. These rankings are then aggregated into average rating. Customers look for product average ratings, to see which product deserve their attention. Customers tend to ignore products with low average rating. Similar to text comments, average ratings facilitate in converting browsers into buyers, and increasing customer loyalty to the site.

\subsection{SMS}

Like emails, recommendations can also be delivered directly to customers through sms. Customers are likely to purchase items if the sms has products based on their own shopping habits and preferences. These days people are always on the 
move. They are not always in front of a computer. So the problem is how to contact these people. A cell phone is one thing which one can count on a person always having with them. That's why sms based recommendations is the most effective way to reach out to customers. Text messaging is an effective way to alert customers of new products, upcoming deals and daily coupons.

\subsection{Display Similar Items Having Power of 99}

Retailers have many products priced just below a round number, because psychologically a \$ 999 product feels more like a deal than $\$ 1000$ product, even if the difference is only one dollar. Recommender systems often implement strategy of 99 , in which similar items (priced below a round number) for which a customer is looking for are displayed.

\section{RECOMMENDATION TECHNIQUES AND THEIR LIMITATIONS}

\subsection{Collaborative Recommendation}

The idea behind the collaborative recommendation approach is to make use of information about the past activities or the views of an existing user community for forecasting which items the current user will most probably like or be interested in. Collaborative recommendations are generally categorized into two categories: (i) User based collaborative recommendation, and (ii) Item based collaborative recommendation.

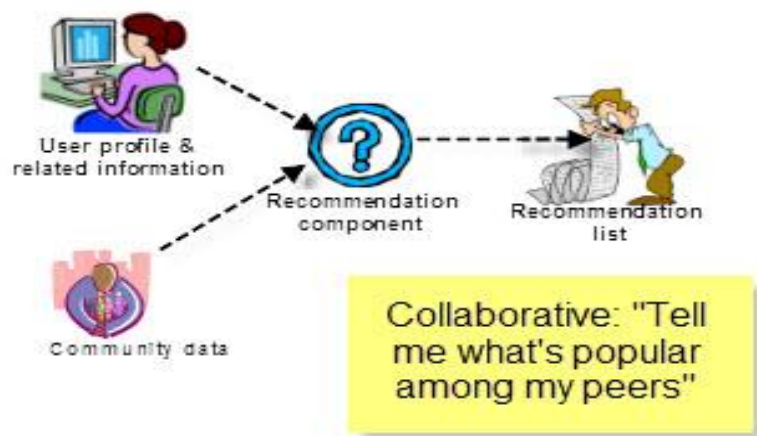

Figure 1: Collaborative recommender system

Figure 1 shows the inputs required and output produced by a collaborative recommender system. The idea behind the user based collaborative recommendation is simply as follows: given a ratings database and the ID of the current (active) user as an input, identify other users (sometimes referred to as peer users or nearest neighbors) that had similar preferences to those of the active user in the past. A rating prediction is computed for every item that the current user has not seen yet. The ratings computed for an item depends on the ratings given by the peer users for that item. The underlying assumptions of such methods are that (a) if users had similar tastes in the past they will have similar tastes in the future and (b) user preferences remain stable and consistent over time.

The idea behind the item based collaborative recommendation is to compute predictions using the similarity between items and not the similarity between users. As explained earlier because the need to scan a vast number of potential neighbors makes it impossible to compute predictions in real time. Therefore e-commerce sites often implement item-based recommendation, which is more appropriate for offline preprocessing and thus allows for the computation of recommendations in real time even for a very large rating matrix.

\section{Limitations of Collaborative Recommendations}

- Cold start problem: This problem is a well known issue in collaborative recommendation systems. There is very less information about new users, which results in an inability to draw inferences to recommend items to users.

- New item problem: New items are added regularly to recommender systems. Collaborative systems rely solely on user's preferences to make recommendations. The recommender system would not be able to draw rating inferences about a new item until the new item is rated by a considerable number of users.

- Scalability: As the numbers of users and items grow, collaborative filtering algorithms will suffer grave scalability problems. A demand for higher scalability of a collaborative filtering system must be fulfilled as many systems need to respond immediately to online requirements and make recommendations for all users.

\subsection{Content-based Recommendation}

Content-based recommendation methods are based on a profile of the user's preference and a description of the item. In a content-based recommender system, items are described by keywords. A user profile is maintained to indicate the type of item a user likes. Those items which are similar to items liked by the user in the past are recommended by these algorithms. Though such an approach must rely on additional information about items and user preferences but it does not require the existence of a large user community or a rating history that is, recommendation lists can be generated even if there is only one single user. Content based recommendation requires upholding an explicit list of features for each item (also often called attributes, characteristics, or item profile). For a book recommender (Figure 2), one could, for instance, use the genre, the author's name, the publisher, or anything else that describes the item and store this information in a database system. When the user's preferences are described in terms of his or her interests using exactly this set of features, the recommendation task consists of matching item characteristics and user preferences.

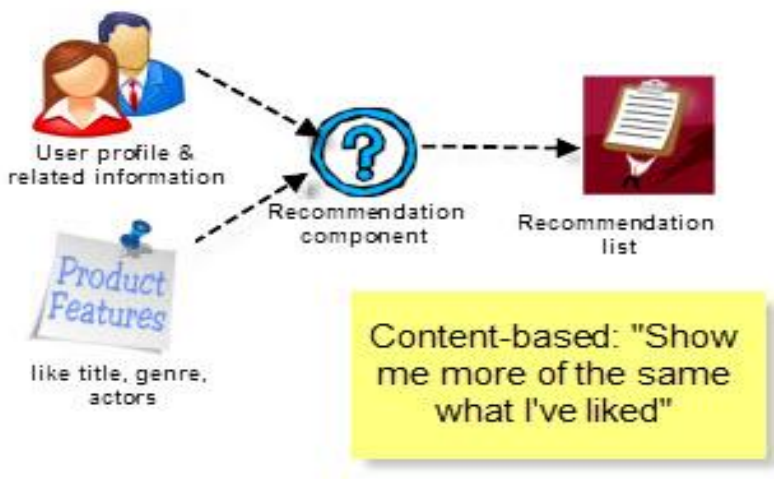

Figure 2: Content-based recommender system

\section{Limitations of Content-based Recommendations}

- Limited content analysis: Content-based recommendation techniques are limited by the characteristics that are associated with the objects that these systems recommend. In order to have sufficient characteristics, the characteristics can be assigned to 
items manually or can be parsed automatically by a program. Automatic characteristic/feature extractions are harder to apply to the multimedia data such as images, video and audio streams. Another problem associated with limited content analysis is that, two items are indistinguishable if they are represented by the same set of characteristics.

- Acquiring ratings: The cold-start problem, which we discussed for collaborative systems, also exists in a slightly different form for content-based recommendation methods. Although content-based techniques do not require a large user community, they require at least an initial set of ratings from the user, typically a set of explicit "like" and "dislike" statements.

\subsection{Hybrid Recommendation}

Collaborative filtering exploits a specific type of information (i.e., item ratings) from a user model together with community data to derive recommendations, whereas content-based approaches rely on product features and textual descriptions. Each of these basic approaches has its pros and cons - for instance, the ability to handle data sparsity, cold-start problems and overspecialization. Hybrid approaches can be implemented in many ways, such as by making content-based and collaborative-based predictions separately and then combining them or by adding content-based capabilities to a collaborative-based approach or by unifying the approaches into one model. Figure 3 shows the basic outline of a hybrid recommender system.

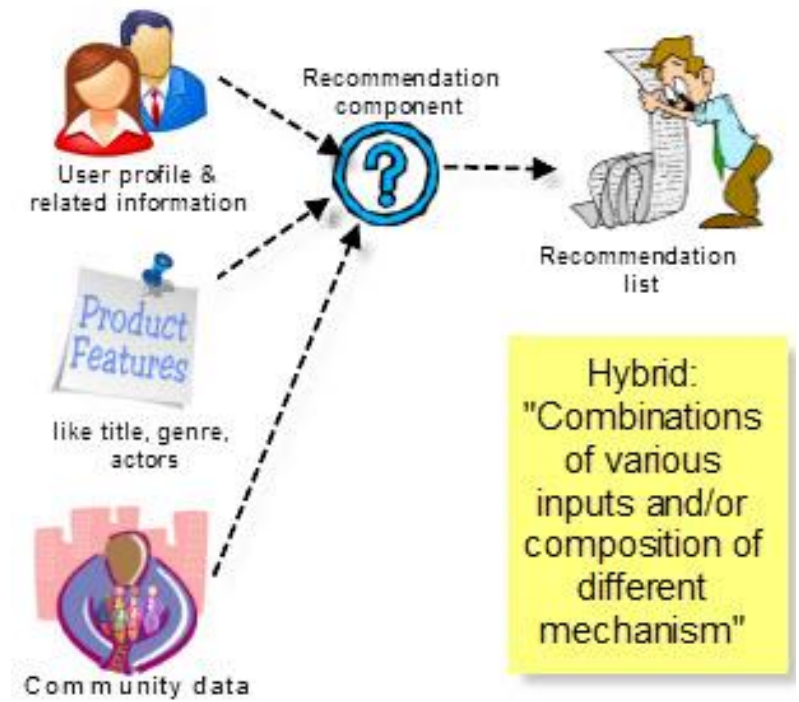

Figure 3: Hybrid recommender system

\section{CONCLUSION}

Recommender systems are turning up everywhere, from movies to news to travel and dating websites. Recommender applications address a variety of e-commerce business needs. Recommender systems are creating value for both customers and e-commerce websites. Recommender systems allow businesses to leverage their customer history to create more personalized experiences for their customers. Customers prefer those recommenders that "know them best", that is the one that can serve them most effectively, recommending the right products. Their advantages seem to prevail over their drawbacks. Recommender systems are out there: watching, learning \& recommending. This paper gives a brief idea about the adaptation of recommender systems in social networks and conent-based systems and concepts related to recommender systems like collaborative recommendation, content-based recommendation and hybrid recommendation.

\section{REFERENCES}

[1] Rich, E. User Modeling via Stereotypes. Cognitive Science, vol. 3, no.4, pp. 329-354, 1979.

[2] Goldberg, D., D. Nichols, B. M. Oki, and D. Terry. Using collaborative filtering to weave an information tapestry. Communications of the ACM, vol. 35, no. 12, pp. 61-70, 1992.

[3] Konstan, J. A., B. N. Miller, D. Maltz, J. L. Herlocker, L. R. Gordon, and J. Riedl. GroupLens: Applying collaborative filtering to Usenet news. Communications of the ACM, vol. 40, no. 3, pp. 77-87, 1997.

[4] Resnick, P., N. Iakovou, M. Sushak, P. Bergstrom, and J. Riedl. GroupLens: An open architecture for collaborative filtering of netnews. In Proceedings of the ACM conference on Computer Supported Cooperative Work, pp. 175- 186, New York, NY, USA, 1994.

[5] Hill, W., L. Stead, M. Rosenstein, and G. Furnas. Recommending and evaluating choices in a virtual community of use. In Proceedings of CHI'95 SIGCHI Conference on Human Factors in Computing Systems, pp. 194-201, ACM press, New York, NY, USA, 1995.

[6] Shardanand and P. Maes. Social information filtering: Algorithms for automating 'word of mouth'. In Proceedings of SIGCHI Conference on Human Factors in Computing Systems, pp. 210-217, ACM press, NY, USA, 1995

[7] Goldberg, K., T. Roeder, D. Gupta and C. Perkins. Eigen taste: A constant time collaborative filtering algorithm. Information Retrieval Journal, vol. 4, no. 2, pp. 133-151, July 2001.

[8] Breese, J. S., D. Heckerman, and C. Kadie. Empirical analysis of predictive algorithms for collaborative filtering. In Proceedings of the Fourteenth Conference on Uncertainty in Artificial Intelligence, pp. 43- 52, Morgan Kaufmann Publishers, San Francisco, CA, USA, 1998.

[9] Delgado, J. and N. Ishii. Memory-based weightedmajority prediction for recommender systems. In ACM SIGIR'99 Workshop on Recommender Systems: Algorithms and Evaluation, ACM press, New York, 1999.

[10] Nakamura, A. and N. Abe. Collaborative filtering using weighted majority prediction algorithms. In Proceedings of the $15^{\text {th }}$ International Conference on Machine Learning (ICML'98), pp. 395- 403, Morgan Kaufmann Publishers,CA, USA, 1998.

[11] Hofmann, T. Collaborative Filtering via Gaussian Probabilistic Latent Semantic Analysis. In Proc. of the $26^{\text {th }}$ Annual International ACM SIGIR Conference, pp. 259-266, ACM press, New York, NY, USA, 2003.

[12] Pavlov, D. and D. Pennock. A Maximum Entropy Approach To Collaborative Filtering in Dynamic, Sparse, High-Dimensional Domains. In Proceedings of the 16th Annual Conference on Neural Information Processing Systems (NIPS'02), pp. 1441-1448, MIT Press, Cambridge, Mass, USA, 2002. 
[13] Ungar, L. H., and D. P. Foster. Clustering methods for collaborative filtering. In Proceedings of the workshop on Recommender Systems, pp. 112-125, AAAI Press, Madison, Wisconsin, USA, 1998.

[14] Billsus, D. and M. Pazzani. Learning collaborative information filters. In International Conference on Machine Learning (ICML'98), pp. 46-54, Morgan Kaufmann Publishers, San Francisco, CA, USA, 1998.

[15] Dempster, A. P., N. Laird and D. B. Rubin. Maximum likelihood from incomplete data via the EM algorithm, Journal of Royal Statistical Society, Series B, vol. 39, no. 1, pp. 1- 38, 1977.

[16] Salton, G. Automatic Text Processing. Addison-Wesley Longman Publishing co., Boston, MA, USA, 1989.

[17] Balabanovic, M. and Y. Shoham. Fab: Content-based, collaborative recommendation. Communications of the ACM, vol. 40, no. 3, pp. 66-72, 1997.

[18] Pazzani, M. and D. Billsus. Learning and revising user profiles: The identification of interesting web sites. Machine learning, vol. 27, no. 3, pp. 313-331, 1997.

[19] Billsus, D. \& M. Pazzani. A Hybrid User Model for News Story Classification. In Proceedings of $7^{\text {th }}$ International Conference on User Modelling, pp. 99-108, Banff, Canada, 1999.

[20] Billsus, D. and M. Pazzani. User modeling for adaptive news access. User Modeling and User-Adapted Interaction, vol. 10, no. 2-3, pp. 147-180, Kluwer Academic Publishers Hingham, MA, USA, 2000.

[21] Tran, T. and R. Cohen. Hybrid Recommender Systems for Electronic Commerce. In Knowledge-Based Electronic Markets, Papers from AAAI Workshop, Technical Report WS-00-04, pp. 78-83, AAAI Press, 2000.

[22] Pazzani, M. A framework for collaborative, contentbased and demographic filtering. Artificial Intelligence Review, vol. 13. No. 5-6, pp. 393-408, December 1999.

[23] Basu, C., H. Hirsh, and W. Cohen. Recommendation as classification: Using social and content-based information in recommendation. In Proceesdings of the $15^{\text {th }}$ National Conference on Artificial Intelligence (AAAI'98), pp. 714- 720, Madison, Wis, USA, July 1998.

[24] Popescul, A., L. H. Ungar, D. M. Pennock, and S. Lawrence. Probabilistic Models for Unified Collaborative and Content-Based Recommendation in Sparse-Data Environments. In Proceedings of the 17th Conf. on Uncertainty in Artificial Intelligence (UAI'01), pp. 437-444, Seattle, WA, 2001.

[25] Schein, A. I., A. Popescul, L. H. Ungar, and D. M. Pennock. Methods and Metrics for cold-start recommendations. In Proceedings of the $25^{\text {th }}$ Annual International ACM SIGIR Conference on Research andDevelopment in Information Retrieval (SIGIR '02), pp. 253-260, ACM press, New York, NY, USA, 2002.

[26] Melville, P., R. J. Mooney, and R. Nagarajan. ContentBoosted Collaborative Filtering for Improved Recommendations. In Proceedings of the Eighteenth National Conference on Artificial Intelligence, Edmonton, pp. 187-192, AAAI press, Menlo Park, CA, USA, 2002

[27] Soboroff, I. and C. Nicholas. Combining content and collaboration in text filtering. In 43 IJCAI'99 Workshop: Machine Learning for Information Filtering, Stockholm, Sweden, August 1999.

[28] Schafer, J.A. Konstan \& J. Riedl. E-Commerce Recommendation Applications. Data Mining \& Knowledge Discovery, vol. 5, no. 1-2, pp. 115-152, 2001.

[29] Forte, D. Shoppers want email with personalized product recommendations, www.listrak.com/about/newsevents/shoppers-want-more-emails-with-personalizedproduct-recommendations, January 15, 2014 [viewed on April 2, 2014].

[30] Myles Anderson. Local Consumer Survey, http://www.brightlocal.com/2010/11/29/local-consumerreview-survey-2010-part-1/, November 29, 2010 [viewed on April 2, 2014]. 